\title{
Brain Regions Mediating Flexible Rule Use during Development
}

\author{
Eveline A. Crone, ${ }^{1,3}$ Sarah E. Donohue, ${ }^{1}$ Ryan Honomichl, ${ }^{1}$ Carter Wendelken, ${ }^{1}$ and Silvia A. Bunge ${ }^{1,2}$ \\ ${ }^{1}$ Center for Mind and Brain and ${ }^{2}$ Department of Psychology, University of California, Davis, California 95616, and ${ }^{3}$ Department of Psychology, Leiden \\ University, 2333AK, Leiden, The Netherlands
}

\begin{abstract}
During development, children improve at retrieving and using rules to guide their behavior and at flexibly switching between these rules. In this study, we used functional magnetic resonance imaging to examine the changes in brain function associated with developmental changes in flexible rule use. Three age groups (8-12,13-17, and 18-25 years) performed a task in which they were cued to respond to target stimuli on the basis of simple task rules. Bivalent target stimuli were associated with different responses, depending on the rule, whereas univalent target stimuli were associated with fixed responses. The comparison of bivalent and univalent trials enabled the identification of regions modulated by demands on rule representation. The comparison of rule-switch and rule-repetition trials enabled the identification of regions involved in rule switching. We have used this task previously in adults and have shown that ventrolateral prefrontal cortex (VLPFC) and the (pre)-supplementary motor area (pre-SMA/SMA) have dissociable roles in task-switching, such that VLPFC is associated most closely with rule representation, and pre-SMA/SMA is associated with suppression of the previous task set (Crone et al., 2006a). Based on behavioral data in children (Crone et al., 2004), we had predicted that regions associated with task-set suppression would show mature patterns of activation earlier in development than regions associated with rule representation. Indeed, we found an adult-like pattern of activation in pre-SMA/SMA by adolescence, whereas the pattern of VLPFC activation differed among children, adolescents, and adults. These findings suggest that two components of task-switching-rule retrieval and task-set suppression—follow distinct neurodevelopmental trajectories.
\end{abstract}

Key words: rules; task-switching; cognitive control; prefrontal cortex; children; development

\section{Introduction}

Between infancy and late adolescence, children develop the ability to exert control over their thoughts and actions, a capacity often referred to as executive function or cognitive control (Diamond, 2002; Zelazo, 2004). A critical component of cognitive control is the effective use of contextually appropriate task rules, or prescribed guides for action. Rule representation is conceptualized as the ability to retrieve, maintain, and use the currently relevant rule(s). Effective rule use hinges on the ability to represent rules as well as the ability to switch flexibly between rules (Wylie and Allport, 2000; Monsell, 2003).

Neuroscientific research suggests that rule representation and rule switching are neurally dissociable (Crone et al., 2006a). Studies in nonhuman primates (Murray et al., 2000; Passingham et al., 2000; Bussey et al., 2002), as well as event-related functional magnetic resonance imaging (fMRI) studies in humans (Toni et al., 1999; Brass and von Cramon, 2002; Brass et al., 2003; Bunge et al., 2003; Bunge, 2004), have implicated ventrolateral prefrontal cortex (VLPFC) as having a role in the development of the ability to

Received May 22, 2006; revised Sept. 13, 2006; accepted Sept. 15, 2006

This research was supported by National Science Foundation Grant 00448844 and a Talent Grant from The Netherlands Organization for Scientific Research (E.A.C.).

Correspondence should be addressed to Eveline A. Crone, Department of Developmental Psychology, Leiden University, Wassenaarseweg 52, 2333AK, Leiden, The Netherlands. E-mail: ecrone@fsw.leidenuniv.nl. DOI:10.1523/JNEUROSCI.2165-06.2006

Copyright $\odot 2006$ Society for Neuroscience ～0270-6474/06/2611239-09\$15.00/0 learn and use rules to perform a task. In contrast, transcranial magnetic stimulation and neuroimaging experiments have shown the importance of medial PFC [specifically, the presupplementary motor area (pre-SMA/SMA)] and superior parietal cortex in rule switching (Sohn et al., 2000; Rushworth et al., 2002, 2004; Braver et al., 2003; Woodward et al., 2006). Finally, in a neuroimaging study with adult participants, we showed that VLPFC and pre-SMA/SMA had dissociable patterns of activation, with VLPFC playing a greater role in rule representation and pre-SMA/SMA playing a greater role in rule switching (Crone et al., 2006a). Superior and inferior parietal cortices were engaged in both functions.

It is known that PFC undergoes structural changes throughout childhood and adolescence (Reiss et al., 1996; Kanemura et al., 2003; Gogtay et al., 2004). These changes are thought to be critical for cognitive maturation (Stuss, 1992; Diamond, 2002; Luna and Sweeney, 2004; Casey et al., 2005). Changes in cortical thickness occur earlier for dorsal medial PFC (including preSMA/SMA) than for VLPFC (Sowell et al., 2004), suggesting that functions that rely on pre-SMA/SMA reach mature levels earlier than functions that rely on VLPFC. Indeed, several behavioral studies have suggested that rule representation and rule switching follow separate developmental trajectories (Cepeda et al., 2001; Crone et al., 2004). In previous behavioral research, we showed that the ability to maintain a rule on-line (i.e., rule representation) continued to develop into adolescence, whereas the ability 
to switch to a new rule (i.e., rule switching) showed the adult pattern by the age of 12 years (Crone et al., 2004).

These previous behavioral findings, together with our neuroimaging findings (Crone et al., 2006a), led to the prediction that we would observe protracted developmental changes into adolescence in the function of VLPFC, a region associated with bivalent rule representation (Brass et al., 2003; Bunge et al., 2003). The comparison of bivalent versus univalent rule use provided us with an index of the capability to represent rules, which includes the ability to retrieve, maintain, and use currently relevant rules while suppressing interference from competing rules.

We further predicted developmental changes in the function of pre-SMA/SMA, a region associated with rule switching (Rushworth et al., 2002, 2004). In contrast to VLPFC, the pattern of pre-SMA/SMA engagement across several task conditions was expected to be adult-like by adolescence (Cepeda et al., 2001; Crone et al., 2004). To test these predictions, we examined age differences in VLPFC and pre-SMA/SMA, as well as superior parietal cortex involvement in rule representation and rule switching across three age groups: children, 8-12 years; adolescents, 13-17 years; and young adults, $18-25$ years.

\section{Materials and Methods}

Participants. Sixty-three volunteers, ranging in age from 8 to 25 years, were recruited through local advertisements and from the University of California at Davis. One adult was excluded because of equipment malfunction; seven participants aged 8-12 and one aged 17 were excluded because of excessive movement ( $>3 \mathrm{~mm}$ across scans). Thus, 54 healthy, right-handed, native English-speaking volunteers were included in the study. Participants were subdivided into three age groups as follows: 17 volunteers aged $8-12$ years (mean age: $10.1 ; 9$ girls, 8 boys); 17 volunteers aged $13-17$ years (mean age: $15.0 ; 11$ girls, 6 boys); and 20 volunteers aged $18-25$ years (mean age: $19.6 ; 12$ women, 8 men). The division of age groups was based on previous behavioral research (Huizinga et al., 2006; Crone et al., 2006a), and served the purpose of making the current results comparable with earlier research. A $\chi^{2}$ analysis confirmed that the gender distribution did not differ significantly among age groups $\left(X^{2}(2)=\right.$ $1.01 ; p=0.60)$. Additional analyses indicated that there was no main effect of gender for accuracy, reaction times, or region-of-interest (ROI) activation, and no interactions involved gender; all $p>0.10$. The adult data have been published separately, as evidence for the functional dissociation between rule representation and rule switching (Crone et al., 2006a).

Behavioral assessment. Children participated in a separate behavioral testing session that took place $1-7 \mathrm{~d}$ before scanning. Participants aged $18-25$ participated in a testing session right before the scan session. IQ scores were estimated with the Raven Standard Progressive Matrices (RSPM) test. Mean IQ scores were as follows: 123 for the 8-12 year olds; 118 for the 13-17 year olds; and 122 for the 18-25 year olds. These differences were not significant $(F<1)$. Participants were screened for psychiatric conditions with the Child Behavior Checklist (Achenbach, 1991) for participants aged $8-17$ years or with the Symptom Checklist-Revised for those aged 18-25 years. All participants had scores within 1 SD of the mean of a normative standardized sample.

Experimental design. The task used in the scanner was designed to manipulate demands on rule representation and flexible rule switching. The rule representation manipulation is operationalized as the difference in behavioral performance and neural activity associated with the use of task rules that assign the same stimuli to different responses ("bivalent" rules) or task rules that assign nonoverlapping sets of stimuli to the required responses ("univalent" rules) (Brass et al., 2003; Crone et al., 2006a). Participants in the present study were asked to respond to targets that could be bivalent (associated with different responses depending on which of two rules is currently relevant) or univalent (associated with fixed responses). The comparison of rule-switch and rule-repetition trials in an event-related fMRI paradigm enabled the identification of regions involved in rule switching (Meiran et al., 2000; Monsell, 2003).

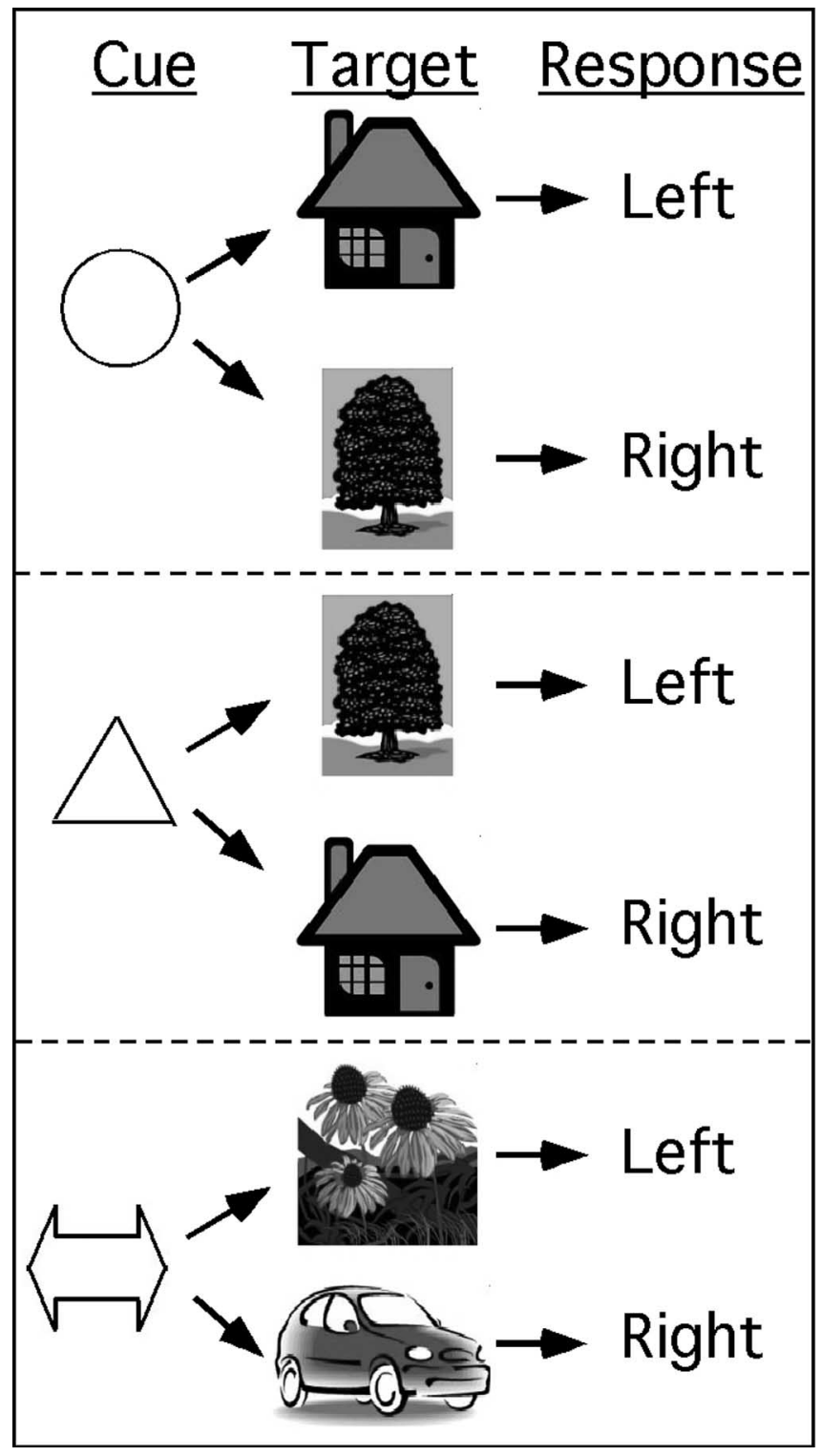

Figure 1. Display of rule types. During scanning, participants viewed an instructional cue for $1 \mathrm{~s}$. After a $0.5 \mathrm{~s}$ delay, the target stimulus was presented for $2.5 \mathrm{~s}$. The target required a left- or right-button response, depending on the relevant $S-R$ mapping learned before scanning.

In addition to the event-related scans, all participants performed the three rules in a blocked task design. Age differences in performance and neural activation were expected to be minimal in the blocked task but substantial in the mixed task. Thus, the blocked task was considered a point of comparison for differences in behavior and activation across age groups in the mixed task; however, direct comparisons between levels of activation on the blocked and mixed tasks is ill-advised because of several potential confounds: (1) learning effects, because blocked scans always occurred first; (2) attention effects related to the unpredictable sequence of conditions in the mixed task; and (3) differences in modeled signal strength when conditions are interleaved versus blocked. As such, our measure of rule-switching focused on switch and repetition trials in the mixed scans, rather than on differences between blocked and mixed scans.

During training, participants learned to associate each of three visual cues with a set of stimulus-response (S-R) associations (Fig. 1). The task involved a visual cue that instructed the participant which rule to use, followed by a target stimulus that required a left- or right-button response. Participants used the index and middle fingers of their left hand to respond. The cue could be a circle, triangle, or bidirectional arrow. The 
circle cue could be followed by a house or a tree, and participants were instructed to respond to the house with a left-button press and to the tree with a right-button press. The triangle cue could also be followed by a house or a tree, but for this cue the S-R mapping was reversed; the house was associated with a right-button response and the tree with a leftbutton response. The arrow cue could be followed by a flower or a car; the former was associated with a left-button response and the latter with a right-button response. Thus, the circle and triangle rules were considered bivalent rules, because the appropriate responses to the targets depended on which rule was currently in effect. In contrast, the arrow rule was considered a univalent rule, because each target was associated with a specific response. We decided to use one instead of two univalent rules to keep the number of stimulus elements constant (two pictures associated with the univalent rule, and two pictures associated with the bivalent rules).

Trials were $4 \mathrm{~s}$ long and had the following structure: a cue was presented for $1 \mathrm{~s}$ followed by a $0.5 \mathrm{~s}$ delay (blank screen) and then by the target. The presentation of the target was response terminated, as long as responses occurred within $2.5 \mathrm{~s}$. After a response, the target was replaced by a fixation cross for the remainder of the trial. Before the scan, participants practiced each of the rules separately in blocks of 15 trials per rule and then performed one practice block of 90 trials in which the rules were intermixed.

Data acquisition. Children were familiarized with the scanner environment through the use of a mock scanner on the same day as the behavioral testing session. Immediately before scanning, children and adults were trained on the fMRI task. During training, participants received 15 practice trials of each type, followed by one mixed block of 90 trials. In the first scan, participants performed a block of 90 trials ( 30 trials per rule) in which the rules were presented in $60 \mathrm{~s}$ blocks of 15 trials each, separated by $20 \mathrm{~s}$ of fixation. The blocks were ordered randomly, and all participants were given the same block order. Participants then performed three event-related scans. They performed a total of 270 experimental trials in which the three rules were intermixed. The event-related scans included 90 trials per rule, distributed equally across the three scans, for a total of 180 bivalent trials and 90 univalent trials. Each participant performed 60 bivalent rule repetitions, 60 bivalent-to-bivalent rule switches, 60 univalent-to-bivalent switches, 30 univalent rule repetitions, and 60 bivalent-to-univalent switches. An equal number of trials of each type required left-button and right-button responses. The order of trial types within each scan was determined with an optimal sequencing program designed to maximize the efficiency of recovery of the blood oxygenation level-dependent response (Dale, 1999). Periods of fixation lasting between 2 and $8 \mathrm{~s}$, jittered in increments of $2 \mathrm{~s}$, were interleaved with the experimental trials as determined by the optimization program. Each of the four functional scans lasted $8 \mathrm{~min}$.

Scanning was performed with a standard whole-head coil on a 1.5 tesla GE scanner at the University of California at Davis Imaging Research Center. Functional data were acquired with a gradient-echo echo-planar pulse sequence (repetition time $=2 \mathrm{~s}$; echo time $=40 \mathrm{~ms} ; 24$ axial slices; $3.44 \times 3.44 \times 5 \mathrm{~mm} ; 0 \mathrm{~mm}$ inter-slice gap; 235 vol per run). Before each scan, four volumes were discarded to allow for longitudinal relaxation time (T1)-equilibration effects. High-resolution T1-weighted anatomical images were collected. Head motion was restricted with a pillow and foam inserts that surrounded the head. Visual stimuli were projected onto a screen that was viewed through a mirror.

fMRI data analysis. Data were preprocessed with SPM2 (Wellcome Trust Functional Imaging Laboratory, Department of Cognitive Neurology, London, UK). Images were corrected for differences in timing of slice acquisition followed by rigid-body motion correction. Structural and functional volumes were spatially normalized to $\mathrm{T} 1$ and echo planar imaging templates, respectively. The normalization algorithm used a 12parameter affine transformation together with a nonlinear transformation involving cosine basis functions and resampled the volumes to $3 \mathrm{~mm}$ cubic voxels. The brain template used for normalization was based on the MNI305 stereotaxic space (Cocosco et al., 1997), an approximation of Talairach space (Talairach and Tourneaux, 1988). This procedure has been validated for use in children aged 6 and older (Burgund et al., 2002; Kang et al., 2003) and has the advantage that regions of activation can be compared directly between age groups. Functional volumes were spatially smoothed with an $8 \mathrm{~mm}$ full-width at half-maximum isotropic Gaussian kernel.

Statistical analyses were performed on individual participants' data with the general linear model in SPM2. fMRI time series data were modeled by a series of impulses convolved with a canonical hemodynamic response function. Each trial was modeled as an event, time-locked to the onset of the cue period. Error trials were modeled separately and were excluded from the analyses. The resulting functions were used as covariates in a general linear model, along with a basic set of cosine functions that high-pass filtered the data, and as a covariate for session effects. The least-squares parameter estimates of the height of the best-fitting canonical hemodynamic response function for each condition were used in pairwise contrasts. The resulting contrast images, computed on a subject-by-subject basis, were submitted to group analyses. At the group level, contrasts between conditions were computed by performing onetailed $t$ tests on these images, treating subjects as a random effect. Taskrelated responses were reported if they consisted of at least 10 contiguous voxels that exceeded an uncorrected threshold of $p<0.001$.

ROI analyses were performed with the Marsbar toolbox for use with SPM2 (Brett et al., 2002). Functional regions were masked with predefined anatomical regions. ROIs consisted of all active voxels within a specific Marsbar anatomical ROI. A general contrast was used to generate functional ROIs, namely of all correct trials $>$ fixation, across all participants (based on blocked plus mixed-task results). For all ROI analyses, effects were considered significant at $\alpha=0.017$ (corrected for multiple comparisons across ROIs in VLPFC, pre-SMA/SMA, and parietal cortex). Behavioral and ROI data met the assumptions for Mauchley's test for sphericity and were adjusted with Huynh-Feldt corrections to adjust for inhomogeneity of the variance-covariance matrix.

\section{Results}

\section{Behavioral results}

Performance in the scanner

All age groups performed well on the rule task, but age-related differences in performance were observed for both accuracy and reaction time (RT) (Fig. 2). As expected, these differences were more pronounced for the mixed task than the blocked task. Agerelated changes were observed for both rule representation and rule switching. Age-related changes in rule representation were measured as reductions in rule costs (decrements in performance for bivalent relative to univalent rules). Similarly, age-related changes in rule switching were measured as reductions in switch costs (decrements in performance for rule switch trials relative to rule repetition trials). Consistent with our previous findings (Crone et al., 2004), rule switching performance reached adults levels earlier in development than rule representation performance.

\section{Blocked task}

An age group $\times$ rule type (bivalent, univalent) ANOVA revealed that participants were less accurate on bivalent than univalent trials $\left(F_{(1,54)}=21.33 ; p<0.001\right)$ and that overall error rates decreased with age $\left(F_{(2,54)}=4.64 ; p<0.05\right)$. Post hoc Tukey comparisons showed that 8 - to 12 -year-old participants made more errors than 18-to 25-year-old participants, but that 13 - to 17-year-old participants did not differ from those aged either $8-12$ or $18-25$ years. The ANOVA further showed an interaction between rule type and age group $\left(F_{(2,54)}=3.78 ; p<0.05\right)$. Post hoc comparisons revealed that the difference in accuracy for bivalent relative to univalent rules was significant for all age groups, but that the difference was larger for those aged 8-12 and 13-17 years than for those aged $18-25$ years (age group $\times$ rule type interaction; $F_{(1,38)}=5.93$ and $p<0.05$, and $F_{(1,36)}=4.67$ and $p<0.05$, respectively). The two younger age groups did not differ from each other $(p>0.20)$. Thus, there were small but significant performance differences for bivalent rule use in the blocked 
task, whereby children and adolescents made more errors than adults.

A similar ANOVA for RT revealed a main effect of rule type $\left(F_{(1,54)}=53.63\right.$; $p<0.001$ ), showing that participants responded more slowly to bivalent than to univalent rules. There was also a main effect of age group $\left(F_{(1,54)}=7.58 ; p<\right.$ $0.001)$, showing that participants aged 8-12 and 13-17 years responded more slowly than those aged 18-25 years. RTs for the two younger age groups did not differ from each other; however, the interaction between rule type and age group was not significant $(p>0.30)$. In summary, children and adolescents exhibited a greater rule cost for accuracy than did adults, consistent with previous findings that rule representation undergoes protracted developmental changes (Cepeda et al., 2001; Davidson et al., 2006).

\section{Mixing costs}

The next analysis focused on the behavioral effects of blocked versus mixed presentations of univalent and bivalent rules. The ANOVA for accuracy revealed an interaction among age group, task (blocked vs mixed), and rule type (bivalent vs univalent): $F_{(2,54)}=6.71$; $p<0.001$. As can be seen in Figure 2, the age group $\times$ task interaction was significant for bivalent rules $\left(F_{(2,54)}=6.97 ; p<\right.$ $0.001)$ but not for univalent rules $\left(F_{(2,54)}=2.31 ; p=0.11\right)$. An age group $\times$ task $\times$ rule type interaction was also found for RTs $\left(F_{(2,54)}=8.69 ; p<0.001\right.$. Here, the age group $\times$ task interaction was significant for both univalent rules $\left(F_{(2,54)}=4.20 ; p<0.05\right)$ and bivalent rules $\left(F_{(2,54)}=11.05 ; p<0.001\right)$, but the differences were larger for bivalent rules than for univalent rules. Follow-up analyses showed that all age group $\times$ task interactions were consistent for the comparisons between children and adolescents $(p<0.05)$ and between adolescents and adults $(p<0.05)$, suggesting that the ability to use rules in a mixed task continues to develop in adolescence. The next set of analyses focused on developmental differences in rule representation and rule switching in the mixed task.

\section{Mixed task}

The ANOVAs for RTs and accuracy in the mixed task focused on rule type (bivalent vs univalent) and rule switching (repetitions vs switches). Error trials, as well as trials after an error trial, were excluded from analysis. Rule representation was conceptualized as the cost of responding to bivalent rules relative to univalent rules. Rule switching was conceptualized as the cost of switching to a bivalent rule relative to repeating a bivalent rule. In general, the data showed that participants responded more slowly and less accurately to bivalent compared with univalent rules and to bivalent switches compared with bivalent repetitions. These effects were magnified for the younger age groups, as can be seen in Figure 2.

\section{Rule representation}

The ANOVA for accuracy revealed age-related increases in the ability to control bivalent rules, as indicated by an age group $\times$ rule type interaction $\left(F_{(2,53)}=9.05 ; p<0.001\right)$. Follow-up ANOVAs showed that all age groups made more errors in response to bivalent rules than to univalent rules, but those aged $8-12$ years made disproportionately more errors in response to bivalent rules than did those aged 18-25 years (age group $\times$ rule type interaction for two age groups: $\left.F_{(1,38)}=25.82 ; p<0.05\right)$. The performance of adolescents was intermediate to that of the children and adults: they made disproportionately more errors in response to bivalent trials than did adults $\left(F_{(1,36)}=6.32\right.$; $p<0.05)$ and tended to make fewer such errors than children $\left(F_{(1,36)}=3.5 ; p=0.07\right)$. This pattern of results shows that the ability to respond accurately to bivalent rules in the context of a mixed-task block develops during childhood and adolescence.

A similar ANOVA for RTs also resulted in a significant age group $\times$ rule type interaction $\left(F_{(2,53)}=11.42 ; p<0.001\right)$. Follow-up ANOVAs showed that RT slowing for bivalent relative to univalent rules was significant for all age groups, but the difference was larger for those aged 8-12 and 13-17 years than for those aged $18-25$ years $\left(F_{(1,38)}=22.63, p<0.05\right.$ and $F_{(1,36)}=$ $7.15, p<0.05$, respectively). The age group $\times$ rule type interaction comparing the 8- to 12-year-old group and the 13- to 17year-old group failed to reach significance $(p=0.10)$. These results confirm that flexible use of bivalent rules continues to develop during adolescence.

\section{Rule switching}

In terms of accuracy, there was a significant age group $\times$ rule type $\times$ rule switch interaction $\left(F_{(2,53)}=4.48 ; p<0.001\right)$. This interaction revealed that univalent switch costs (accuracy on univalent switches vs univalent repetitions) were similar across age groups $(F<1)$, whereas the cost of bivalent switching (accuracy on bivalent switches vs bivalent repetitions) decreased with age $\left(F_{(2,53)}=3.04 ; p=0.05\right)$. Post hoc comparisons revealed that the bivalent switch costs were larger for those aged $8-12$ years than for the adults $\left(F_{(1,37)}=6.59 ; p<0.05\right)$, but those aged $13-17$ years did not differ from either the 8- to 12-year-old participants or the adults (both $p$ values $>0.1$ ). Thus, the ability to accurately switch between rules on this task showed a mature pattern at approximately the beginning of adolescence. 

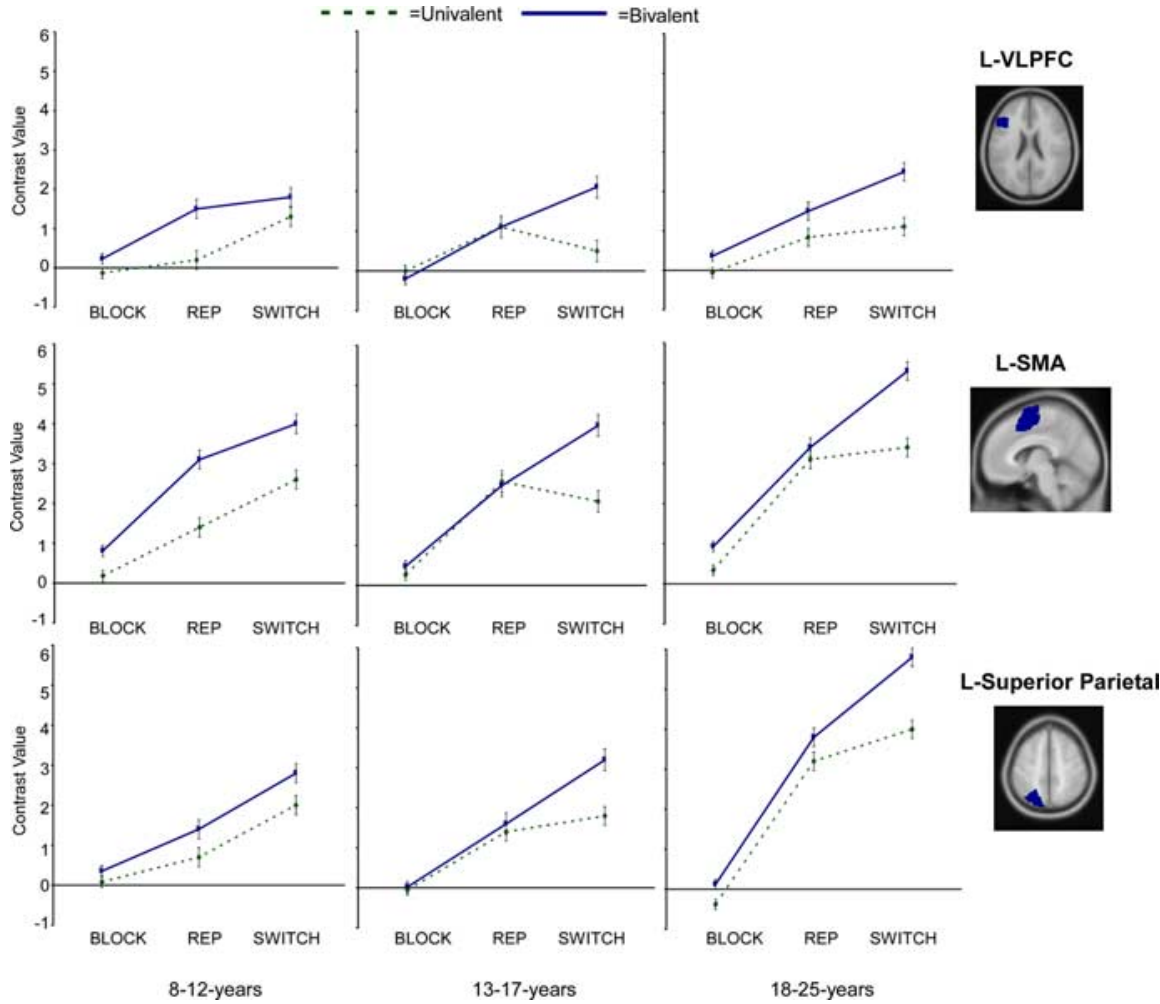

Figure 3. ROI results for left (L-) VLPFC (BA 45; $-42,24,18)$, left pre-SMA/SMA (BA $6 ;-6,3,60)$, and left superior parietal cortex (BA 7; $-24,-66,52)$ for children, adolescents, and adults. BLOCK, Blocked presentation; REP, repetition trials in the mixed block; SWITCH, switch trials in the mixed block. All ROls were identified by the whole-brain contrast of all correct trials relative to fixation, across all participants ( $p<0.001$ uncorrected). Error bars depict an estimate of within-subject SE.

Rule switching also resulted in a slowing of RTs $\left(F_{(1,53)}=\right.$ $66.86 ; p<0.001)$. An interaction between rule switching and rule type $\left(F_{(1,53)}=28.78 ; p<0.001\right)$ revealed that, although the RT decrease was significant for both conditions, this differences was larger for bivalent rule switching (bivalent switches-bivalent repetitions) $\left(F_{(1,52)}=50.20 ; p<0.001\right)$ compared with univalent rule switching (univalent switches-univalent repetitions) $\left(F_{(1,52)}=\right.$ $11.35 ; p<0.001)$. Importantly, the age group $\times$ rule switch interaction was not significant $(p>0.15)$. Thus, age differences in switching were reflected only in terms of accuracy.

Previous studies have shown that switch effects can be affected by whether the response switches or repeats from one trial to the next. We considered the possibility that developmental differences in rule switching were related to age differences associated with response switches versus repetitions (Crone et al., 2004; Davidson et al., 2006); however, none of the reported switch effects were altered by response repetition-switching for either the behavioral results or the brain imaging results. These results are consistent with our previous findings in adults (Crone et al., 2006a).

\section{Brain imaging results: ROIs}

ROI analyses were performed for bilateral VLPFC (BA 45), bilateral pre-SMA/SMA (BA 6), and bilateral superior parietal cortex (BA 7) (Fig. 3). Age group $\times$ rule type ANOVAs were performed for the blocked task, and age group $\times$ rule type $\times$ task switch ANOVAs were performed for the mixed task. Significant interactions were followed by post hoc analyses.

\section{Blocked task}

A main effect of rule type (bivalent $>$ univalent) was observed in both left and right pre-SMA/SMA $\left(F_{(1,51)}=9.9, p<0.005\right.$, and
$F_{(1,51)}=9.8, p<0.005$, respectively). No such effect was observed for either VLPFC or superior parietal cortex (all $F$ values $<1$ ). Importantly, no interactions with age group were observed in the blocked task for any of the ROIs (all $F$ values $<1$ ). Thus, there were no systematic differences between age groups in terms of modulation by rule type in these ROIs when the rules were performed in separate blocks.

\section{Mixing costs}

To examine the effects of blocked versus mixed presentation of rules on neural activity, we performed age group $\times$ task (blocked vs mixed) $\times$ rule type ANOVAs for each ROI. As can be seen in Figure 3, bivalent rule use resulted in more activation than univalent rule use in the mixed task relative to the blocked task for VLPFC $\left(F_{(1,55)}=28.09 ; p<0.001\right)$, pre-SMA/ SMA $\left(F_{(1,55)}=19.12 ; p<0.001\right)$, and parietal cortex $\left(F_{(1,55)}=21.20 ; p<0.001\right)$, but these effects were not different for the three age groups (no significant interactions with age group).

\section{Mixed task}

\section{VLPFC}

The analysis for left VLPFC revealed a significant three-way interaction among age group, rule type, and rule switch $\left(F_{(2,51)}=\right.$ 8.2; $p<0.001$ ), and left and right VLPFC activation did not differ from each other (interactions with hemisphere: $F$ values $<1$ ). Age-related differences were observed across all three age groups in terms of the pattern of activation in VLPFC (Fig. 3). As reported previously (Crone et al., 2006a), adults exhibited greater VLPFC activation for bivalent than for univalent trials, even when rules were repeated $\left(F_{(1,19)}=5.2 ; p<0.05\right)$, showing that this region is sensitive to rule representation in general. In contrast, children exhibited greater VLPFC activation for switch trials, with an unexpected pattern of greater responsiveness to switching on univalent than on bivalent trials (age $\times$ switch interaction: $\left.F_{(2,50)}=4.7 ; p<0.05\right)$. Adolescents also differed from adults in terms of VLPFC activation, in that they exhibited an effect of rule type only on switch trials (age $\times$ rule type $\times$ switch interaction: $\left.F_{(2,50)}=4.5 ; p<0.05\right)$. These data show that children and adolescents did not engage VLPFC in this rule task in the same way as adults.

\section{Pre-SMA/SMA}

An age group $\times$ rule type $\times$ switch interaction was also observed for left pre-SMA/SMA $\left(F_{(2,51)}=3.2 ; p<0.05\right)$, and this pattern did not differ significantly from that of right pre-SMA/SMA (all $F$ values $<1$ ). Post hoc comparisons revealed that those aged $8-12$ years showed a different pattern of activation compared with those aged 13-17 years and adults, but the latter two age groups did not differ from one another $(F<1)$. Separate analyses showed rule type $\times$ switch interactions for adults $\left(F_{(1,19)}=7.1\right.$; $p<0.05)$ and adolescents aged $13-17$ years $\left(F_{(1,16)}=18.1 ; p<\right.$ $0.005)$. These interactions show that adults and adolescents recruited pre-SMA/SMA more strongly for bivalent switch $>$ repetition trials $\left(F_{(1,19)}=35.0, p<0.001\right.$, and $F_{(1,16)}=18.3, p<$ 
0.001, respectively) but not for univalent switch $>$ repetition trials (both $F$ values $<1$ ). In contrast, the rule type $\times$ switch interaction was not significant for children aged 8-12 years $\left(F_{(1,16)}=0.14 ; p=0.70\right)$, such that children showed a switch effect in this medial PFC region equally for univalent and bivalent trials.

Post hoc comparisons revealed that children aged 8-12 years engaged pre-SMA/SMA similarly to adults and adolescents for bivalent switching $>$ repetition (age $\times$ switch: $F_{(2,50)}=1.48 ; p=$ $0.24)$. In contrast, children aged $8-12$ years showed disproportionately greater activation in pre-SMA/SMA for bivalent $>$ univalent trials on repetition trials, a pattern that was not observed for adolescents and adults (rule $\times$ age interaction: $F_{(2,50)}=3.94$; $p<0.05)$. Although there was a trend toward a larger univalent switch cost for children, this interaction did not reach significance $\left(F_{(2,50)}=2.6 ; p=0.10\right)$. These results suggest that all age groups used pre-SMA/SMA for bivalent rule switching, and those aged $8-12$ years additionally recruited this region for rule representation. Thus, unlike VLPFC, pre-SMA/SMA showed the adult-like pattern of activation on this rule task early in adolescence.

Lateral versus medial PFC contributions to flexible rule use To compare the relative contributions of VLPFC and pre-SMA/ SMA with rule representation and rule switching in each age group, we performed separate region (left VLPFC vs left preSMA/SMA $) \times$ rule type $\times$ switch ANOVAs for each age group. These ANOVAs revealed a three-way interaction for adults $\left(F_{(1,19)}=7.3 ; p<0.05\right)$ but not for adolescents or children (both $F$ values $<1$ ), indicating greater functional differentiation in adulthood. In adolescents and adults, a greater switch effect was observed for bivalent trials in pre-SMA/SMA than in VLPFC (region $\times$ switch: $F_{(1,16)}=5.8, p<0.05$, and $F_{(1,19)}=9.3, p<0.01$, respectively). This effect did not reach significance in children aged $8-12$ years (region $\times$ switch: $F_{(1,16)}=3.36 ; p=0.09$ ). In contrast, children aged $8-12$ years showed a greater rule effect in pre-SMA/SMA than in VLPFC (region $\times$ rule: $F_{(1,16)}=11.7 ; p<$ $0.001)$. This effect was not observed in either of the older age groups (both $F$ values $<1$ ).

\section{Inferior frontal junction}

A region that has been associated with rule implementation is the inferior frontal junction (IFJ) (at the junction between BA 6, 9, and 44) (Brass et al., 2005). We drew a sphere of this region (radius 10, coordinates: $-38,2,32$ ) based on previous work by Brass and colleagues (2005) to examine whether this region showed a pattern that was dissociable from VLPFC and pre-SMA. A region (VLPFC vs IFJ) $\times$ age group $\times$ rule type $\times$ switch ANOVA revealed no differences between the patterns of activity in VLPFC and IFJ (all $F$ values $<1$ ). A separate age group $\times$ rule type $\times$ switch ANOVA for IFJ resulted in a significant three-way interaction $\left(F_{(2,50)}=3.7 ; p<0.05\right)$, with a pattern of activity similar to that of VLPFC. In contrast, a similar analysis for preSMA/SMA versus IFJ showed that pre-SMA/SMA is more active when switching than IFJ (contrast values 3.6 vs 2.7 , respectively) relative to repetition trials (contrast values 1.9 vs 1.4 , respectively) (region $\times$ switch: $\left.F_{(1,50)}=8.7 ; p<0.005\right)$. Thus, the IFJ appeared to play a role similar to that of VLPFC on this task and underwent similar developmental changes.

\section{Superior parietal cortex}

The analysis for left superior parietal cortex revealed effects of rule type $\left(F_{(1,51)}=38.0 ; p<0.001\right)$ and rule switching $\left(F_{(1,51)}=\right.$ $38.3 ; p<0.001)$. Although there was a general increase in supe- rior parietal activation among adults relative to participants aged $13-17$ and $8-12$ years (main effect of age group: $F_{(2,50)}=10.49$; $p<0.001$; followed by Tukey B post hoc comparisons), there were no age differences for rule type or task switch effects (all interactions: $F$ values $<1$ ). The pattern for left superior parietal cortex did not differ significantly from right superior parietal cortex (interactions with hemisphere: $F$ values $<1$ ). Thus, despite the general age-related increase in activation, all age groups used bilateral superior parietal cortex in the same way for rule representation and rule switching.

\section{Brain-behavior correlations}

We performed regression analyses on the ROI data to test for effects of IQ and age on behavioral rule costs and switch costs. Additionally, we performed regression analyses on the ROI data to test for brain-behavior correlations. All correlations were nonsignificant; however, the changes that occur over childhood are most likely nonlinear and therefore may not have been revealed by our tests for linear relations. Further research is required to characterize the shape of the function relating performance and brain activation over development in regions associated with flexible rule-guided behavior.

\section{Whole-brain analysis}

Although the main focus of this paper is on regions in specific frontal and parietal regions, we report below the results of relevant whole-brain comparisons.

\section{Rule representation in the blocked task}

Consistent with the behavioral findings, there was a weak effect of rule type on brain activation in the blocked task. For adults, a comparison between bivalent and univalent rules in the blocked task revealed activation in left superior frontal gyrus (BA 6) and left anterior cingulate cortex (BA 32). Adolescents aged 13-17 years showed activation in a single region in right inferior frontal gyrus (BA 47). Children aged 8-12 years, in contrast, showed a widespread pattern of activation for this comparison (supplemental Table 1, available at www.jneurosci.org as supplemental material).

\section{Rule representation and rule switching in mixed task}

In contrast to the blocked task, the mixed task revealed a robust effect of rule type on brain activation (supplemental Table 2, available at www.jneurosci.org as supplemental material). For the bivalent $>$ univalent comparison, children activated a set of regions similar to those that we had identified in the adults (Crone et al., 2006a), including bilateral insula, bilateral VLPFC (BA 44/47), left middle frontal gyrus (BA 9), pre-SMA/SMA (BA 6), left putamen, and bilateral inferior parietal cortex (BA 40) and superior parietal cortex (BA 7). For the bivalent $>$ univalent comparison, children as young as $8-12$ years of age recruited many of the same regions as those aged 18-25 years (Fig. 4).

\section{Discussion}

\section{Rule representation: blocked task}

Consistent with previous behavioral research (Cepeda et al., 2001; Davidson et al., 2006), we found developmental changes in rule representation in the blocked task, with children and adolescents having more difficulty using bivalent rules relative to univalent rules than adults. The brain imaging contrasts show that children aged 8-12 years implement rules in a more effortful manner than adults on the blocked trials. For adults and adolescents, the effects of rule type on brain activation (bivalent $>$ 


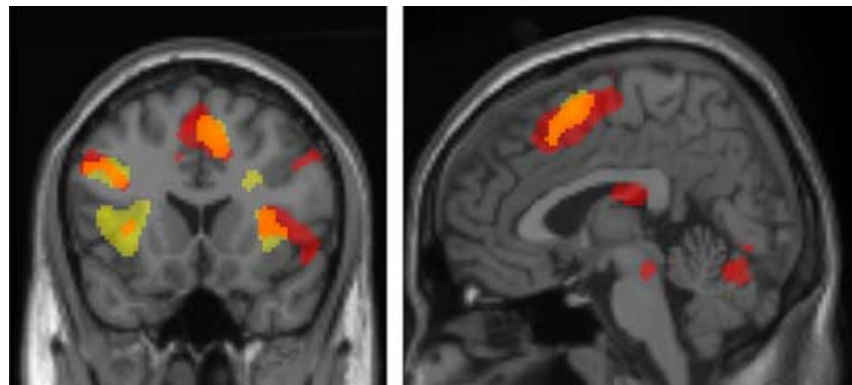

Figure 4. Overlap in activation for children aged 8-12 years and adults for the contrast bivalent rules $>$ univalent rules in the mixed task. Activation in children is in red; activation in adults is in yellow. The overlap between children and adults is in orange. Rule-related activation was observed for both age groups in left VLPFC (BA 44, 45), bilateral insula (BA 13), and preSMA/SMA (BA 6).

univalent rules) were large in the mixed task but minimal in the blocked task. Notably, in adults, one of the two regions that did show an effect of rule representation in the blocked task was the dorsal premotor cortex (BA 6), near a region that has been implicated in rule implementation for both newly learned and well learned rules (Amiez et al., 2006). In contrast, 8- to 12-year-old children exhibited widespread activation for the bivalent $>$ univalent contrast in the blocked design. This finding suggests that children aged $8-12$ years engaged in more effortful rule representation than adolescents and adults, even when the same rule was repeated over and over [see also Davidson et al. (2006)].

\section{Rule representation: mixed task}

Age-related performance differences were much larger in the mixed task, consistent with previous studies in which children and adolescents showed large mixing costs (Cepeda et al., 2001). Children and adolescents also showed larger response costs when responding to mixed bivalent rules relative to mixed univalent rules. In a previous behavioral study, this developmental trend was also found for uniquely mapped bivalent rules, for which the response side rather than the target was bivalent (Davidson et al., 2006); thus, the response cost to bivalent rules most likely reflects a valence effect with respect to targets as well as locations. Together, these findings show that developmental changes in the performance of school-aged children are most evident in a dynamically changing task environment (Huizinga et al., 2006).

Like adults, children and adolescents engaged VLPFC and superior parietal cortex more strongly when responding to bivalent versus univalent rules; however, adolescents and children showed a pattern that deviated from that of adults in VLPFC, a region consistently associated with rule representation in adults and in nonhuman primates (Bunge et al., 2003; Bunge, 2004). This slow developmental trajectory is consistent with previous behavioral studies, which have shown that rule representation becomes more efficient during childhood, because children improve in their ability to represent multiple task rules (Zelazo, 2004). In summary, the behavioral results show elevated rule costs for children aged 8-12 years and adolescents aged 13-17 years relative to adults, and the fMRI data show an immature pattern of activation in VLPFC among children aged 8-12 years and adolescents aged 13-17 years relative to adults.

\section{Task-set suppression}

Consistent with the existing literature on task-switching (Wylie and Allport, 2000; Logan and Bundesen, 2003; Monsell, 2003), participants were slower and less accurate when switching to a new rule than when repeating the rule used on the preceding trial. As in previous studies, this switch cost was larger for bivalent rules than for univalent rules (Meiran et al., 2000) and for children aged 8-12 years than for adolescents or adults (Cepeda et al., 2001; Davidson et al., 2006).

When switching between bivalent task rules, all age groups showed increased activation in pre-SMA/SMA relative to rule repetition or to the blocked presentation. Adults and adolescents recruited this region more for bivalent switching than for univalent switching, consistent with previous research in adults indicating that pre-SMA/SMA is important for task-switching when the task rules are associated with bivalent S-R mappings (Rushworth et al., 2004; Woodward et al., 2006). In contrast, 8- to 12-year-old children activated pre-SMA/SMA not only for rule switching but also for rule representation, as revealed by increased activation also for bivalent rule repetitions relative to univalent repetitions. It is possible that the activation pattern in pre-SMA in children is caused by their failure to treat bivalent repetition trials like univalent repetition trials in the mixed task context. Adults and adolescents engage pre-SMA similarly for univalent and bivalent repetition trials, which may lead to relatively reduced response times to bivalent repetition trials in adults compared with children. In summary, the behavioral results point to elevated switch costs among 8- to 12-year-old children relative to adolescents and adults, consistent with previous behavioral results (Cepeda et al., 2001; Davidson et al., 2006; Kray et al., 2004), and the fMRI data show an immature pattern of activation in pre-SMA/SMA among 8- to 12-year-old children relative to the older age groups.

\section{Separate developmental trajectories for rule representation} and task-set suppression

Developmental fMRI studies of working memory and cognitive control show age-related increases in activation within brain regions linked to task performance in adults as well as age-related decreases within regions that are not critical for performance in adults (Bunge et al., 2002a; Casey et al., 2002; Klingberg et al., 2002; Durston and Casey, 2005; Brown et al., 2006; Durston et al., 2006). In this study, we focused not on age-related differences in level of activation but rather on differences in the pattern of activation across conditions (see also Crone et al., 2006a). This approach reveals that children, adolescents, and adults all recruited VLPFC for rule representation, but in different ways. It further reveals that all three age groups engaged pre-SMA/SMA for rule switching, but that children aged $8-12$ years additionally recruited this region for rule representation.

These results suggest that separate developmental trajectories of two cognitive control functions (rule representation and rule switching) are tied to separate trajectories or brain regions mediating these functions in adults (VLPFC vs pre-SMA/SMA); however, it will be important to determine whether extensive practice on the task reduces or abolishes these group differences. Finally, the current results are important in showing that for some control functions (e.g., rule representation), changes in brain activation are observed also in adolescence, consistent with previous functional (Luna et al., 2001) and structural brain imaging studies (Gogtay et al., 2004), which show that changes in brain function take place throughout adolescence.

Superior parietal cortex was sensitive to both rule representation (bivalent $>$ univalent) and task-switching (switch $>$ repetition), consistent with previous findings showing that this region is generally active when there is a need to control sets of S-R associations (Brass and von Cramon, 2004; Bunge, 2004; Crone et 
al., 2006a). Although we observed increased activation levels in superior parietal cortex across the three age groups, this region contributed to the task rules in the same way in children, adolescents, and adults. Thus, the ability to represent sets of possible responses in parietal cortex (Sohn et al., 2000; Bunge et al., 2002b; Braver et al., 2003) is already present by middle childhood.

One caveat of this study was that cue processing was necessary for the bivalent rules but not for the univalent rules; however, participants in these studies generally use information about the upcoming rule to prepare for the possible response that needs to be made, not only when rule switches are unpredictable, as they were in this study, but even in predictable switch tasks (Monsell, 2003). Moreover, even if it were the case that participants ignored the univalent cue and waited for the stimulus to appear, they would still have to use the stimulus as an instructional cue to determine which response to make. Nonetheless, it is possible that adults and children have used different strategies to perform the task. The effects of strategy training should be examined in future research.

In conclusion, this study shows that brain regions associated with flexible rule use contribute in different ways to this cognitive ability across childhood, adolescence, and adulthood. Furthermore, regions associated with putatively distinct components of rule use exhibit functionally mature patterns of activation at different ages. This latter finding strengthens the claim that rule representation and rule switching are dissociable components of task-switching (Crone et al., 2006a). Two additional limitations should be noted here. First, cross-sectional comparisons of participants in different age groups provide only a coarse indicator of developmental change. Longitudinal studies should be undertaken to confirm that the age effects observed in cross-sectional studies are observed within-subject (Durston et al., 2006). Second, it is possible for a brain region to show adult-like activation patterns in one task but not in another (Crone et al., 2006b). The regions implicated in this research can contribute to several cognitive functions by association with different brain networks, and one network may be mature even when another network is not yet mature. By further characterizing neurodevelopmental changes in cognitive control processes within subjects and across a range of tasks, we hope to better understand the neural mechanisms underlying the development of the human mind.

\section{References}

Achenbach TM (1991) Manual for the child behavior checklist/4-18 and 1991 profile. Burlington, VT: University of Vermont.

Amiez C, Kostopoulos P, Champod AS, Petrides M (2006) Local morphology predicts functional organization of the dorsal premotor region in the human brain. J Neurosci 26:2724-2731.

Brass M, von Cramon DY (2002) The role of the frontal cortex in task preparation. Cereb Cortex 12:908-914.

Brass M, von Cramon DY (2004) Selection for cognitive control: a functional magnetic resonance imaging study on the selection of task-relevant information. J Neurosci 24:8847-8852.

Brass M, Ruge H, Meiran N, Rubin O, Koch I, Zysset S, Prinz W, von Cramon DY (2003) When the same response has different meanings: recoding the response meaning in the lateral prefrontal cortex. NeuroImage 20:1026-1031.

Brass M, Derrfuss J, Forstmann B, von Cramon DY (2005) The role of the inferior frontal junction area in cognitive control. Trends Cogn Sci 9:314-316.

Braver TS, Reynolds JR, Donaldson DI (2003) Neural mechanisms of transient and sustained cognitive control during task switching. Neuron 39:713-726.

Brett M, Anton JL, Valabregue R, Poline JB (2002) Region of interest analysis using an SPM toolbox. The Eighth International Conference on Functional Mapping of the Human Brain, Sendai, Japan, June.
Brown TT, Petersen SE, Schlaggar BL (2006) Does human functional brain organization shift from diffuse to focal with development? Dev Sci 9:9-11.

Bunge SA (2004) How we use rules to select actions: a review of evidence from cognitive neuroscience. Cogn Affect Behav Neurosci 4:564-579.

Bunge SA, Dudukovic NM, Thomason ME, Vaidya CJ, Gabrieli JD (2002a) Immature frontal lobe contributions to cognitive control in children: evidence from fMRI. Neuron 33:301-311.

Bunge SA, Hazeltine E, Scanlon MD, Rosen AC, Gabrieli JD (2002b) Dissociable contributions of prefrontal and parietal cortices to response selection. NeuroImage 17:1562-1571.

Bunge SA, Kahn I, Wallis JD, Miller EK, Wagner AD (2003) Neural circuits subserving the retrieval and maintenance of abstract rules. J Neurophysiol 90:3419-3428.

Burgund ED, Kang HC, Kelly JE, Buckner RL, Snyder AZ, Petersen SE, Schlaggar BL (2002) The feasibility of a common stereotactic space for children and adults in fMRI studies of development. NeuroImage 17:184-200.

Bussey TJ, Wise SP, Murray EA (2002) Interaction of ventral and orbital prefrontal cortex with inferotemporal cortex in conditional visuomotor learning. Behav Neurosci 116:703-715.

Casey BJ, Tottenham N, Fossella J (2002) Clinical, imaging, lesion, and genetic approaches toward a model of cognitive control. Dev Psychobiol 40:237-254.

Casey BJ, Tottenham N, Liston C, Durston S (2005) Imaging the developing brain: what have we learned about cognitive development? Trends Cogn Sci 9:104-110.

Cepeda NJ, Kramer AF, Gonzalez de Sather JC (2001) Changes in executive control across the life span: examination of task-switching performance. Dev Psychol 37:715-730.

Cocosco CA, Kollokian V, Kwan RKS, Evans AC (1997) Brain web: online interface to a 3D MRI simulated brain database. NeuroImage 5:S452.

Crone EA, Ridderinkhof KR, Worm M, Somsen RJ, van der Molen MW (2004) Switching between spatial stimulus-response mappings: a developmental study of cognitive flexibility. Dev Sci 7:443-455.

Crone EA, Wendelken C, Donohue SE, Bunge SA (2006a) Neural evidence for dissociable components of task-switching. Cereb Cortex 16:475-486.

Crone EA, Wendelken C, Donohue SE, Van Leijenhorst L, Bunge SA (2006b) Neurocognitive development of the ability to manipulate information in working memory. Proc Natl Acad Sci USA 103:9315-9320.

Dale AM (1999) Optimal experimental design for event-related fMRI. Hum Brain Mapp 8:109-114.

Davidson MC, Amso D, Anderson LC, Diamond A (2006) Development of cognitive control and executive functions from 4 to 13 years: evidence from manipulations of memory, inhibition, and task switching. Neuropsychologia 44:2037-2078.

Diamond A (2002) Normal development of prefrontal cortex from birth to young adulthood: cognitive functions, anatomy and biochemistry. In: Principles of frontal lobe function (Stuss DT, Knight RT, eds), pp 466503. London: Oxford UP.

Durston S, Casey BJ (2005) What have we learned about cognitive development from neuroimaging? Neuropsychologia 44:2149-2157.

Durston S, Davidson MC, Tottenham N, Galvan A, Spicer J, Fossella JA, Casey BJ (2006) A shift from diffuse to focal cortical activity with development. Dev Sci 9:1-8.

Gogtay N, Giedd JN, Lusk L, Hayashi KM, Greenstein D, Vaituzis AC, Nugent III TF, Herman DH, Clasen LS, Toga AW, Rapoport JL, Thompson PM (2004) Dynamic mapping of human cortical development during childhood through early adulthood. Proc Natl Acad Sci USA 101:8174-8179.

Huizinga M, Dolan CV, van der Molen MW (2006) Age-related change in executive function: developmental trends and a latent variable analysis. Neuropsychologia 44:2017-2036.

Kanemura H, Aihara M, Aoki S, Araki T, Nakazawa S (2003) Development of the prefrontal lobe in infants and children: a three-dimensional magnetic resonance volumetric study. Brain Dev 25:195-199.

Kang HC, Burgund ED, Lugar HM, Petersen SE, Schlaggar BL (2003) Comparison of functional activation foci in children and adults using a common stereotactic space. NeuroImage 19:16-28.

Klingberg T, Forssberg H, Westerberg H (2002) Increased brain activity in frontal and parietal cortex underlies the development of visuospatial working memory capacity during childhood. J Cogn Neurosci 14:1-10.

Kray J, Eber J, Lindenberger U (2004) Age differences in executive function- 
ing across the lifespan: the role of verbalization in task preparation. Acta Psychol (Amst) 115:143-165.

Logan GD, Bundesen C (2003) Clever homunculus: is there an endogenous act of control in the explicit task-cuing procedure? J Exp Psychol Hum Percept Perform 29:575-599.

Luna B, Sweeney JA (2004) The emergence of collaborative brain function: fMRI studies of the development of response inhibition. Ann NY Acad Sci 1021:296-309.

Luna B, Thulborn KR, Munoz DP, Merriam EP, Garver KE, Minshew NJ, Keshavan MS, Genovese CR, Eddy WF, Sweeney JA (2001) Maturation of widely distributed brain function subserves cognitive development. NeuroImage 13:786-793.

Meiran N, Chorev Z, Sapir A (2000) Component processes in task switching. Cognit Psychol 41:211-253.

Monsell S (2003) Task switching. Trends Cogn Sci 7:134-140.

Murray EA, Bussey TJ, Wise SP (2000) Role of prefrontal cortex in a network for arbitrary visuomotor mapping. Exp Brain Res 133:114-129.

Passingham RE, Toni I, Rushworth MF (2000) Specialisation within the prefrontal cortex: the ventral prefrontal cortex and associative learning. Exp Brain Res 133:103-113.

Reiss AL, Abrams MT, Singer HS, Ross JL, Denckla MB (1996) Brain development, gender and IQ in children: a volumetric imaging study. Brain 119:1763-1774.

Rushworth MF, Hadland KA, Paus T, Sipila PK (2002) Role of the human medial frontal cortex in task switching: a combined fMRI and TMS study. J Neurophysiol 87:2577-2592.

Rushworth MF, Walton ME, Kennerley SW, Bannerman DM (2004) Action sets and decisions in the medial frontal cortex. Trends Cogn Sci 8:410-417.

Sohn MH, Ursu S, Anderson JR, Stenger VA, Carter CS (2000) Inaugural article: the role of prefrontal cortex and posterior parietal cortex in task switching. Proc Natl Acad Sci USA 97:13448-13453.

Sowell ER, Thompson PM, Leonard CM, Welcome SE, Kan E, Toga AW (2004) Longitudinal mapping of cortical thickness and brain growth in normal children. J Neurosci 24:8223-8231.

Stuss DT (1992) Biological and psychological development of executive functions. Brain Cogn 20:8-23.

Talairach J, Tourneaux P (1988) Co-planar stereotaxic atlas of the human brain. New York: Thieme.

Toni I, Schluter ND, Josephs O, Friston K, Passingham RE (1999) Signal-, set- and movement-related activity in the human brain: an event-related fMRI study. Cereb Cortex 9:35-49.

Woodward TS, Ruff CC, Ngan ET (2006) Short- and long-term changes in anterior cingulate activation during resolution of task-set competition. Brain Res 1068:161-169.

Wylie G, Allport A (2000) Task switching and the measurement of "switch costs". Psychol Res 63:212-233.

Zelazo PD (2004) The development of conscious control in childhood. Trends Cogn Sci 8:12-17. 